\title{
LICHEN PLANUS IN A HUSBAND AND WIFE
}

\author{
SAMUEL FELDMAN, M.D.
}

Adjunct Attending, Bronx Hospital, Department of Dermatology and Syphilis; Chief of Clinic, Bronx, Lebanon, and Beth David Hospital Dispensaries

NEW YORK

Montgomery, in 1919, reports an instance of lichen planus in two brothers and reviews the literature on the subject. In all, there were twenty-five such instances reported, including his own case. From the extremely small number of cases of lichen planus in several members of one family, he argues that it cannot be due to a family predisposition. In the same article, he says that lichen planus bears a pronounced resemblance to the great microbic diseases, syphilis and leprosy. The fact that the lichens are beneficially influenced by arsenic and mercury, taken internally, the same drugs that are curative in syphilis, leads one to think that there may be some, even if a remote, relation between the causative factors of the two diseases. The idea of a similar etiology in the two diseases, however, is immediately dispelled, when we take into consideration the evident infectious nature of the one and the isolated character of the other disease. I fail to find a single instance of lichen planus in a husband and wife reported in the literature. I believe that this extremely rare occurrence is more than a mere coincidence and that it may help to throw some light on the hitherto unknown cause of lichen planus.

\section{REPORT OF CASES}

I. H., aged 41, a tailor, born in Russia, had eleven months previously noticed little papules on the backs of both hands and on the front of the forearms. The eruption was extremely itchy. Later he noticed the same condition on the penis and scrotum and, to a lesser extent, on both legs. About eight months previously he began to experience a slight burning sensation of the buccal mucosa. White streaks and patches appeared in that location and on the tongue. On examination the patient was found to be a well nourished, well built man. There was no evidence of nervousness and the digestion was good at the time of the examination. He complained of only slight discomfort due to the itching. There were a few pigmented areas on the forearms, penis and scrotum, marking the location of former active lesions. There were a few typical lichen planus papules and several minute patches on the anterior surface of each leg. On the buccal mucosa of both cheeks there were numerous white, shiny papules, and streaks running in a radial direction forward. On the tongue, there was one large patch, pearly white in color. It covered about one third of the surface of the dorsum of the latter organ. Besides the patch, there were a number of white, shiny papules and small lines.

About three months previously, or approximately eight months after the appearance of the disease in the husband, the wife began to feel a roughness of the buccal mucosa of the left cheek. She paid no attention to the slight annoy- 
ance caused by it and remained untreated until she came with her husband to the Bronx Hospital dispensary, the middle of June. At that time, white, shiny streaks were seen at the angle of the jaw on the left side. They were arranged radially. A little farther forward, there was a white glistening line located on a level with the edge of the gum. The lesions were raised, and could easily be felt on the surface of the mucosa. Treatment was withheld from the wife for a period of two months during which time a condition similar to the one already present developed on the right side of the mouth. There also appeared a few typical lichen planus papules on both wrists. Wassermann tests made on three different occasions were negative in both patients. The blood and the urine were normal.

\section{SUMMARY AND CONCLUSIONS}

There was a striking similarity in the appearance and distribution of the lesions, and in the course of the disease. There was the same preponderance of mouth lesions, the same mildness and sparseness of skin lesions, and the same comparative freedom from itching in both patients. Ill health could not be an etiologic factor as both husband and wife had been enjoying good health, and they appeared robust and well nourished. There was no question of a neurosis. The lack of a nervous element is well illustrated by the fact that the wife, at least, was so little mindful of her condition that she did not enlist medical aid until three months after the onset of the disease.

Family predisposition is not to be considered, because the two patients were not related by blood. Furthermore, they were born in different parts of the world.

The suggestion of Montgomery that the disease may be caused by an intestinal toxemia is not borne out in this instance, as both patients had enjoyed uninterrupted good health for many years prior to the present attack.

Taking into consideration the facts that they had been living without interruption in conjugal proximity and that the lesions in the wife.made their appearance eight months after the beginning of the disease in the husband, and considering the striking similarity of the course of the disease in both patients, may I not suggest the possibility of an infectious etiology?

909 Kelly Street. 